\title{
Emergence of classical structures from the quantum vacuum
}

\author{
Mainak Mukhopadhyay $\odot,{ }^{1}$ Tanmay Vachaspati $\odot,{ }^{1}$ and George Zahariade ${ }^{1,2}$ \\ ${ }^{1}$ Physics Department, Arizona State University, Tempe, Arizona 85287, USA \\ ${ }^{2}$ Beyond Center for Fundamental Concepts in Science, Arizona State University, \\ Tempe, Arizona 85287, USA
}

(Received 20 April 2020; revised 13 June 2020; accepted 12 August 2020; published 24 September 2020)

\begin{abstract}
After a quantum phase transition, the quantum vacuum can break up to form classical topological defects. We examine this process for scalar field models with $Z_{2}$ symmetry for different quench rates for the phase transition. We find that the number density of kinks at late times universally scales as $C m^{1 / 2} t^{-1 / 2}$, where $m$ is a mass scale in the model and $C \approx 0.22$; it does not depend on the quench timescale in contrast to the Kibble-Zurek scaling for thermal phase transitions. A subleading correction $\propto t^{-3 / 2}$ to the kink density depends on the details of the phase transition.
\end{abstract}

DOI: 10.1103/PhysRevD.102.056021

\section{INTRODUCTION}

Quantum field theories generally contain small quantum excitations around a true vacuum that we call particles and large classical structures called solitons that interpolate between different degenerate vacua. Often the solitons have a topological character and are then also known as topological defects of which kinks, domain walls, strings, and magnetic monopoles are all examples. As first proposed by Kibble [1], these structures can be formed during a phase transition. A more quantitative estimate of their number density formed after a thermal phase transition is given by the Kibble-Zurek proposal [2-8] that has been tested in various systems such as liquid crystals $[9,10]$, superfluids [11-15], superconductors [16-18], and other systems involving liquid crystal light valves [19] and ultracold quantum gases [20], with conflicting conclusions. The Kibble-Zurek proposal is based on imposing a physically motivated cutoff on the growing correlation length prior to the thermal phase transition and then matching the prephase transition correlation length to the postphase transition correlation length. In this paper, we will be concerned with a quantum phase transition and we will solve for the full quantum dynamics relevant to defect formation.

To describe our approach we start with the $\lambda \phi^{4}$ model for a real, scalar field $\phi$,

$$
L=\frac{1}{2}\left(\partial_{\mu} \phi\right)^{2}-\frac{1}{2} m_{2}(t) \phi^{2}-\frac{\lambda}{4} \phi^{4} .
$$

Published by the American Physical Society under the terms of the Creative Commons Attribution 4.0 International license. Further distribution of this work must maintain attribution to the author(s) and the published article's title, journal citation, and DOI. Funded by SCOAP.
To study the production of kinks in this model, we imagine that the mass parameter $m_{2}(t)$ has an externally controlled time dependence,

$$
m_{2}(t)=-m^{2} \tanh \left(\frac{t}{\tau}\right),
$$

where $\tau$ is the "quench timescale." For $t<0$, the model has a unique vacuum at $\phi=0$, while for $t>0$, there are two degenerate vacua $\phi= \pm m / \sqrt{\lambda} \equiv \pm \eta$. In the $t \rightarrow \infty$ limit, where $m_{2}=-m^{2}$, the model has a double well potential and it admits static classical kink and antikink solutions

$$
\phi_{ \pm}(x)= \pm \eta \tanh \left(\frac{m x}{\sqrt{2}}\right)
$$

These nonperturbative solutions interpolate between the two degenerate vacua of the model over a spatial width $\sim 1 / m$. They are topological defects characterized by a topological charge, positive for a kink and negative for an antikink. In fact, the topological charge classifies kinks and antikinks according to the nature of the sign change occurring in the field profile: negative to positive for a kink, and vice versa for an antikink.

Since all we are interested in is changes in the sign of the field, we can simplify our model to eliminate the $\lambda \phi^{4}$ term in (1) (see Fig. 1). Then the free field model

$$
L=\frac{1}{2}\left(\partial_{\mu} \phi\right)^{2}-\frac{1}{2} m_{2}(t) \phi^{2}
$$

also has $Z_{2}$ symmetry that is spontaneously broken after the quench and thus has topological kinks. Without the $\lambda \phi^{4}$ term, the kink height is not stabilized and becomes larger with time. We expect that, for a small enough value of $\lambda$, the 


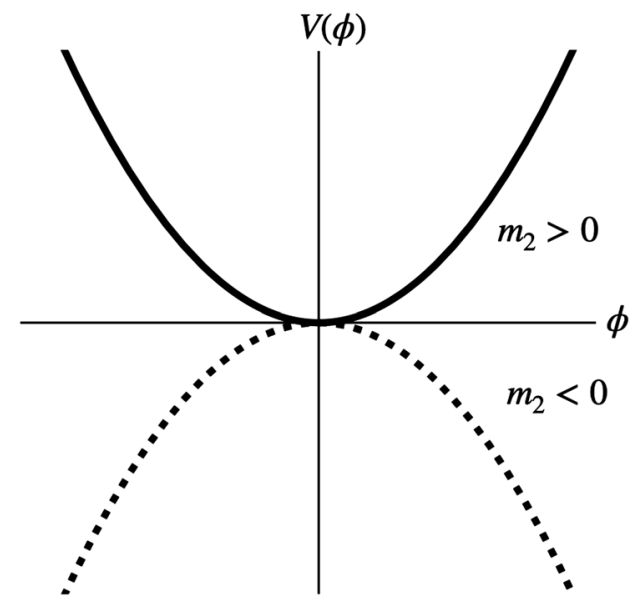

FIG. 1. The field theory potential is an upright quadratic at early times when $m_{2}>0$ and becomes an inverted quadratic after the quench when $m_{2}<0$. The model has a $Z_{2}$ symmetry that is spontaneously broken and hence has kinks.

interaction term does not significantly affect the kink number density. Hence, the model in (4) captures the essential physics of defect formation. ${ }^{1}$ It can serve as the zeroth-order approximation in a perturbative expansion in powers of $\lambda$.

In Sec. II we will solve the quantum field theory problem for the model in (4) in the Schrödinger picture, which is more convenient for the kink number density calculation in Sec. III. Our numerical results for the kink number density and its dependence on various parameters are given in Sec. IV. We conclude and qualitatively describe expectations when $\lambda \neq 0$ in Sec. V. Some technical results are included in the Appendixes.

\section{WAVE FUNCTIONAL}

Setting $\lambda=0$, the problem now is that of a quantum field interacting with a classical background. As discussed in [21-23], the solution to the quantum problem can be written in terms of the solution of a classical problem but in higher dimensions. More specifically, let us discretize a compactified space of size $L$ by $N$ lattice points $i=1, \ldots, N$, with lattice spacing $a=L / N$. The resulting lattice-point-dependent Heisenberg picture field operators $\hat{\phi}_{i}$ describe a set of $N$ quadratically coupled, simple harmonic oscillators, and we can write

$$
\hat{\phi}_{i}=Z_{i j}^{*} \hat{a}_{j}+Z_{i j} \hat{a}_{j}^{\dagger}
$$

where $\hat{a}_{j}$ and $\hat{a}_{j}^{\dagger}$ are the annihilation and creation operators associated with the quantum variable $\hat{\phi}_{i}$ at $t=-\infty$ when

\footnotetext{
${ }^{1}$ One can think of this model as the $\lambda \rightarrow 0, \eta \rightarrow \infty$ limit of the $\lambda \phi^{4}$ model with $m=\eta \sqrt{\lambda}$ held fixed at some chosen finite value.
}

the potential is upright and time independent. The $N \times N$ complex matrix $Z$ satisfies the classical equation

$$
\ddot{Z}+\Omega_{2}(t) Z=0,
$$

where the matrix $\Omega_{2}$ is given by

$$
\left[\Omega_{2}\right]_{i j}= \begin{cases}+2 / a^{2}+m_{2}(t), & i=j \\ -1 / a^{2}, & i=j \pm 1(\bmod N) \\ 0, & \text { otherwise. }\end{cases}
$$

In our particular application, since $m_{2}(t)$ does not depend on the lattice point, all matrices are circulant [i.e., their $(i, j)$ element only depends on $i-j(\bmod N)]$ and the problem is translationally invariant. We can thus diagonalize $Z$ to work in momentum space. This leads to

$$
Z_{j l}=\frac{1}{\sqrt{N}} \sum_{n=1}^{N} c_{n}(t) e^{-i(j-l) 2 \pi n / N},
$$

where the mode coefficients $c_{n}$ satisfy

$$
\ddot{c}_{n}+\left[\frac{4}{a^{2}} \sin ^{2}\left(\frac{\pi n}{N}\right)+m_{2}(t)\right] c_{n}=0 .
$$

The quantum state (in the Heisenberg picture) will be chosen to be the vacuum state long before the phase transition, i.e., at $t=-\infty$ when $m_{2}(t)=+m^{2}$. In practice, this is achieved by choosing an initial time $t_{0} \ll-\tau$ and setting up the following initial conditions for the mode coefficients:

$$
\begin{aligned}
& c_{n}\left(t_{0}\right)=\frac{-i}{\sqrt{2 a N}}\left[\frac{4}{a^{2}} \sin ^{2}\left(\frac{\pi n}{N}\right)+m_{2}\left(t_{0}\right)\right]^{-1 / 4}, \\
& \dot{c}_{n}\left(t_{0}\right)=\frac{1}{\sqrt{2 a N}}\left[\frac{4}{a^{2}} \sin ^{2}\left(\frac{\pi n}{N}\right)+m_{2}\left(t_{0}\right)\right]^{1 / 4} .
\end{aligned}
$$

While it may be easier to solve for the quantum dynamics in momentum space by computing the $c_{n}$ mode coefficients, the kinks are defined as zeros of the field in physical space. It is therefore useful to determine the physical space wave functional $\Psi\left[\left\{\phi_{i}\right\}, t\right]$ for the model in Eq. (4) by solving the corresponding Schrödinger equation,

$$
i \frac{\partial \Psi}{\partial t}=-\frac{1}{2 a} \sum_{i=1}^{N} \frac{\partial^{2} \Psi}{\partial \phi_{i}^{2}}+\frac{a}{2} \phi^{T} \Omega_{2}(t) \phi \Psi
$$

We find 


$$
\begin{aligned}
\Psi\left[\left\{\phi_{i}\right\}, t\right]= & \left(\frac{a}{\pi}\right)^{N / 4} \operatorname{det}\left(\Omega_{2}\left(t_{0}\right)\right)^{1 / 8} \\
& \times \exp \left[-\frac{1}{2} \int_{0}^{t} d t^{\prime} \operatorname{Tr} M\left(t^{\prime}\right)+\frac{i a}{2} \phi^{T} M(t) \phi\right],
\end{aligned}
$$

where $M \equiv \dot{Z} Z^{-1}$ and we have introduced the column vector $\phi$ such that $\phi^{T} \equiv\left(\phi_{1}, \ldots, \phi_{N}\right)$. Using the constraint $Z^{\dagger} \dot{Z}-\dot{Z}^{\dagger} Z=i / a$ [21], this gives a probability distribution for the field

$$
P\left[\left\{\phi_{i}\right\}, t\right]=\frac{1}{\sqrt{\operatorname{det}(2 \pi K)}} e^{-\phi^{T} K^{-1} \phi / 2},
$$

where $K=Z Z^{\dagger}$ is the covariance matrix of the field $\phi$. Notice that $K$ is real and symmetric [21] [as can be verified by using (6) and the initial conditions].

\section{NUMBER DENSITY OF KINKS}

The number density of zeros $\left(n_{Z}\right)$ is obtained by counting the number of sign changes of $\phi$. To compute an explicit formula, we first define the quantum operator

$$
\hat{n}_{Z} \equiv \frac{1}{L} \sum_{j=1}^{N} \frac{1}{4}\left[\operatorname{sgn}\left(\hat{\phi}_{j}\right)-\operatorname{sgn}\left(\hat{\phi}_{j+1}\right)\right]^{2},
$$

where, because of the periodicity of the lattice, $\hat{\phi}_{N+1}=\hat{\phi}_{1}$. The number density of zeros is then simply given by the quantum average of this operator. After using translational invariance and, in particular, the fact that $K^{-1}$ is circulant, it reads (see Appendix A for details),

$$
n_{Z}=\left\langle\hat{n}_{Z}\right\rangle=\frac{N}{2 L}\left[1-\left\langle\operatorname{sgn}\left(\hat{\phi}_{1} \hat{\phi}_{2}\right)\right\rangle\right] .
$$

The expectation value in (16) can now be written as

$$
\begin{aligned}
\left\langle\operatorname{sgn}\left(\hat{\phi}_{1} \hat{\phi}_{2}\right)\right\rangle= & \frac{1}{\sqrt{\operatorname{det}(2 \pi K)}} \\
& \times \sum_{\text {quads }} \int d \phi_{1} \ldots d \phi_{N} \operatorname{sgn}\left(\phi_{1} \phi_{2}\right) e^{-\phi^{T} K^{-1} \phi / 2},
\end{aligned}
$$

where the sum is over the four quadrants in the $\left(\phi_{1}, \phi_{2}\right)$ plane. The coefficient $\operatorname{sgn}\left(\phi_{1} \phi_{2}\right)$ is +1 for quadrants with $\phi_{1} \phi_{2}>0$ and -1 for quadrants with $\phi_{1} \phi_{2}<0$.

Consider the integral in the first quadrant,

$$
I_{1}=\int_{0}^{\infty} d \phi_{1} \int_{0}^{\infty} d \phi_{2} \int_{-\infty}^{\infty} d \phi_{3} \ldots d \phi_{N} e^{-\phi^{\dagger} K^{-1} \phi / 2} .
$$

To perform the integration, we write

$$
K^{-1}=\left(\begin{array}{cc}
A & B \\
B^{T} & C
\end{array}\right)
$$

where $A$ is a $2 \times 2$ matrix, $B$ is a $2 \times(N-2)$ matrix, and $C$ is an $(N-2) \times(N-2)$ matrix. (The matrices $A$ and $C$ are symmetric.) Next, we also introduce the vectors $\chi$ and $\xi$ such that $\chi^{T}=\left(\phi_{1}, \phi_{2}\right)$ and $\xi^{T}=\left(\phi_{3}, \ldots, \phi_{N}\right)$. Then,

$$
\begin{aligned}
\phi^{T} K^{-1} \phi= & \left(\xi+C^{-1} B^{T} \chi\right)^{T} C\left(\xi+C^{-1} B^{T} \chi\right) \\
& +\chi^{T}\left(A-B C^{-1} B^{T}\right) \chi,
\end{aligned}
$$

and we can perform the integration over $\xi$ first using

$$
\int d^{N-2} \xi e^{-\left(\xi+C^{-1} B^{T} \chi\right)^{T} C\left(\xi+C^{-1} B^{T} \chi\right) / 2}=\frac{(2 \pi)^{(N-2) / 2}}{\sqrt{\operatorname{det}(C)}} .
$$

As detailed in Appendix B, we obtain

$$
\begin{aligned}
I_{1} & =\frac{(2 \pi)^{(N-2) / 2}}{\sqrt{\operatorname{det}(C)}} \int_{0}^{\infty} d \phi_{1} \int_{0}^{\infty} d \phi_{2} e^{-\chi^{T} A^{\prime} \chi / 2} \\
& =\frac{(2 \pi)^{(N-2) / 2}}{\sqrt{\operatorname{det}(C) \operatorname{det}\left(A^{\prime}\right)}}\left[\frac{\pi}{2}-\tan ^{-1}\left(\frac{A_{12}^{\prime}}{\sqrt{\operatorname{det}\left(A^{\prime}\right)}}\right)\right],
\end{aligned}
$$

where $A^{\prime}$, the Schur complement of $C$, is defined by $A^{\prime} \equiv A-B C^{-1} B^{T}$.

The integral over the third quadrant in the $\phi_{1} \phi_{2}$ plane, $I_{3}$, is seen to be equal to $I_{1}$ after the change of variables $\chi \rightarrow-\chi$. The integrals over the second and fourth quadrants, $I_{2}$ and $I_{4}$, are similarly equal and are related to $I_{1}$. To see this we note that the integral over the second quadrant reduces to the one over the first quadrant by the transformation $\phi_{1} \rightarrow-\phi_{1}$. This transformation is alternatively implemented by changing $A_{12}^{\prime}$ to $-A_{12}^{\prime}$ and not changing anything else. Hence, the integral over the second quadrant is simply

$$
I_{2}=\frac{(2 \pi)^{(N-2) / 2}}{\sqrt{\operatorname{det}(C) \operatorname{det}\left(A^{\prime}\right)}}\left[\frac{\pi}{2}+\tan ^{-1}\left(\frac{A_{12}^{\prime}}{\sqrt{\operatorname{det}\left(A^{\prime}\right)}}\right)\right] .
$$

Putting together the contributions of all the four quadrants, we get

$$
\left\langle\operatorname{sgn}\left(\hat{\phi}_{1} \hat{\phi}_{2}\right)\right\rangle=-\frac{2}{\pi} \tan ^{-1}\left(\frac{A_{12}^{\prime}}{\sqrt{\operatorname{det}\left(A^{\prime}\right)}}\right),
$$

where we made use of the fact that $A^{\prime}$ is the Schur complement of $C$, which implies

$$
\operatorname{det}\left(A^{\prime}\right) \operatorname{det}(C)=\operatorname{det}\left(K^{-1}\right)=\frac{1}{\operatorname{det}(K)} .
$$


This, in turn, gives us the number density of zeros as

$$
n_{Z}=\frac{N}{2 L}\left[1+\frac{2}{\pi} \tan ^{-1}\left(\frac{A_{12}^{\prime}}{\sqrt{\operatorname{det}\left(A^{\prime}\right)}}\right)\right] .
$$

We can make this formula more explicit by noticing that $\left(A^{\prime}\right)^{-1}$ is equal to the $2 \times 2$ upper left block of the matrix $K$.

This can be seen via a block LDU decomposition of $K^{-1}$ as described in Appendix C. Using (8) we can then write

$$
\left(A^{\prime}\right)^{-1}=K_{2 \times 2}=\alpha \mathbf{1}+\beta \sigma_{x},
$$

where $\sigma_{x}$ is the first Pauli spin matrix and

$$
\alpha=\sum_{n=1}^{N}\left|c_{n}\right|^{2}, \quad \beta=\sum_{n=1}^{N}\left|c_{n}\right|^{2} \cos (2 \pi n / N) .
$$

Therefore,

$$
A^{\prime}=\frac{1}{\alpha^{2}-\beta^{2}}\left(\alpha \mathbf{1}-\beta \sigma_{x}\right)
$$

and

$$
\operatorname{det}\left(A^{\prime}\right)=\frac{1}{\alpha^{2}-\beta^{2}}, \quad A_{12}^{\prime}=\frac{-\beta}{\alpha^{2}-\beta^{2}} .
$$

We now have all the pieces needed to evaluate the number density of zeros in (24), which can be written as

$$
n_{Z}=\frac{N}{2 L}\left[1-\frac{2}{\pi} \theta\right]
$$

where

$$
\sin \theta \equiv \frac{\beta}{\alpha}=\frac{\sum_{n=1}^{N}\left|c_{n}\right|^{2} \cos (2 \pi n / N)}{\sum_{n=1}^{N}\left|c_{n}\right|^{2}}
$$

Not all field zeros, however, correspond to kinks. Some of the zeros simply correspond to field oscillations that come in and out of existence. They are spurious or virtual kinks and we eliminate them from our counting by restricting the summations in (30) to modes that are not oscillating. ${ }^{2}$ So the number density of kinks $n_{K}$ is

$n_{K}=\frac{N}{2 L}\left[1-\frac{2}{\pi} \sin ^{-1}\left(\frac{\sum_{|n| \leq n_{c}}\left|c_{n}\right|^{2} \cos (2 \pi n / N)}{\sum_{|n| \leq n_{c}}\left|c_{n}\right|^{2}}\right)\right]$,

where, as seen in (9), the time-dependent cutoff mode for $t>0$ is defined by

\footnotetext{
${ }^{2}$ This is similar to the situation in inflationary cosmology where only nonoscillating superhorizon modes lead to density perturbations.
}

$$
\sin \left(\frac{\pi n_{c}(t)}{N}\right)=\frac{a}{2} \sqrt{\left|m_{2}(t)\right|}
$$

and $c_{-n} \equiv c_{N-n}$ for $0 \leq n \leq N-1$.

\section{NUMERICAL RESULTS}

We can now numerically solve (9) and use (31) to obtain the number density of kinks as a function of time. The only scale in the problem is the mass, so we work in units of $1 / m$ by setting $m=1$. We also choose $L=6400$ and $N=12800$, which are both large enough to accurately describe the continuum, infinite space limit of the discretized model. Notice that, thanks to the physical cutoff we placed on the mode sums, there are no UV divergences. In Fig. 2 we show our results for several different values of the quench parameter $\tau$. The remarkable feature of this plot is that all the curves have the same late time behavior, which we can determine to be a $t^{-1 / 2}$ power law. In fact, we can take differences for different values of $\tau, \Delta n_{K}\left(t, \tau_{1}, \tau_{2}\right) \equiv$ $n_{K}\left(t, \tau_{1}\right)-n_{K}\left(t, \tau_{2}\right)$ (see Fig. 3), and these follow a $t^{-3 / 2}$ power law. Thus, at late times we can write

$$
n_{K}(t)=C \sqrt{\frac{m}{t}}+\mathcal{O}\left(t^{-3 / 2}\right)
$$

where we get $C \approx 0.22$ from our numerical solution. Note that $C$ is independent of $\tau$. At early times (i.e., immediately after the phase transition), $n_{K}$ increases from zero to a maximum value $\left(n_{K}\right)_{\max }$ within a time $t_{\max }$, before decreasing again. This is to be expected: the phase transition triggers the creation of kinks with randomly distributed positions and velocities, which later start annihilating with each other. In Figs. 4 and 5 we plot $\left(n_{K}\right)_{\max }$ and $t_{\max }$, respectively, as a function of the quench parameter $\tau$. This confirms the intuitive expectation according to which the

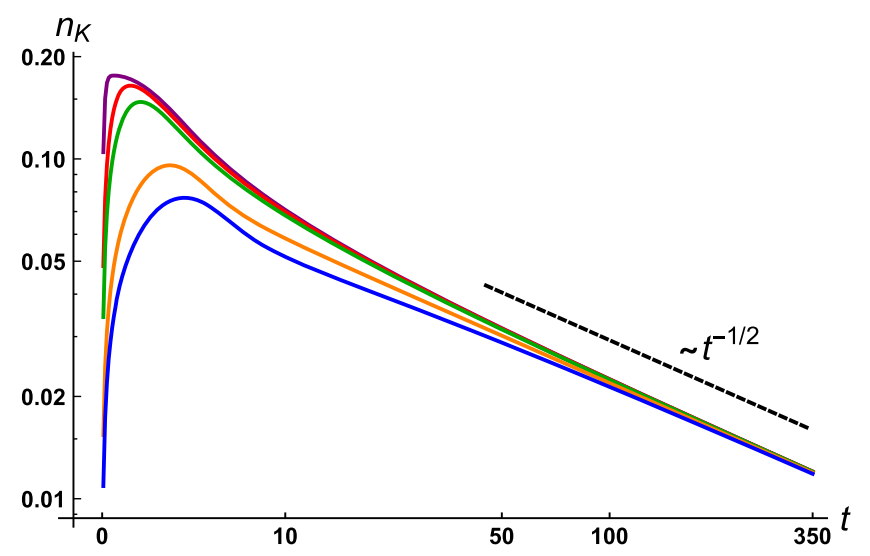

FIG. 2. Log-log plot of $\left\langle n_{K}\right\rangle$ versus time for $\tau=0.1$ (purple, topmost curve), 0.5 (red), 1.0 (green), 5.0 (orange), 10.0 (blue). The black dashed line shows the exhibited power law at late times, i.e., $t^{-1 / 2}$. 


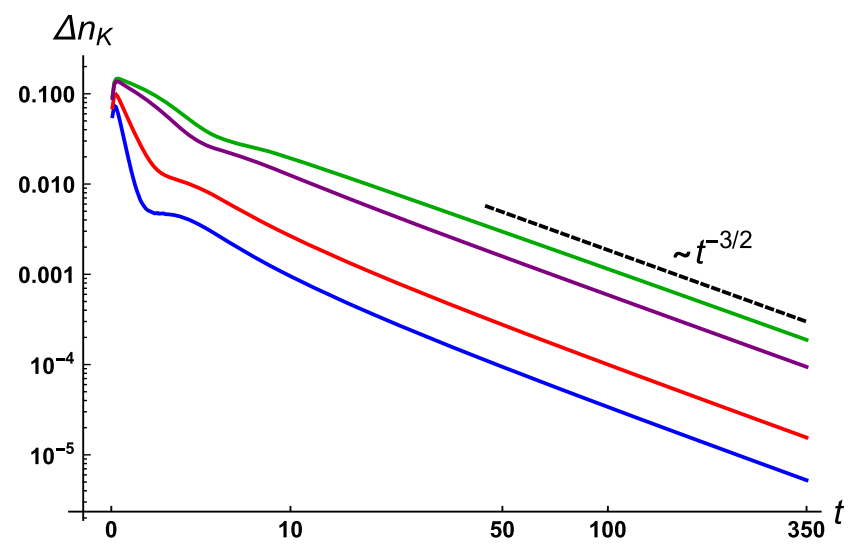

FIG. 3. Differences between the average kink density for different values of $\tau, n_{K}\left(t, \tau_{1}\right)-n_{K}\left(t, \tau_{2}\right)$ versus time, for $\tau_{1}=0.1 ; \tau_{2}=0.5$ (blue, bottom-most curve), 1.0 (red), 5.0 (purple), 10.0 (green). The black dashed line shows the exhibited power law, i.e., $t^{-3 / 2}$.

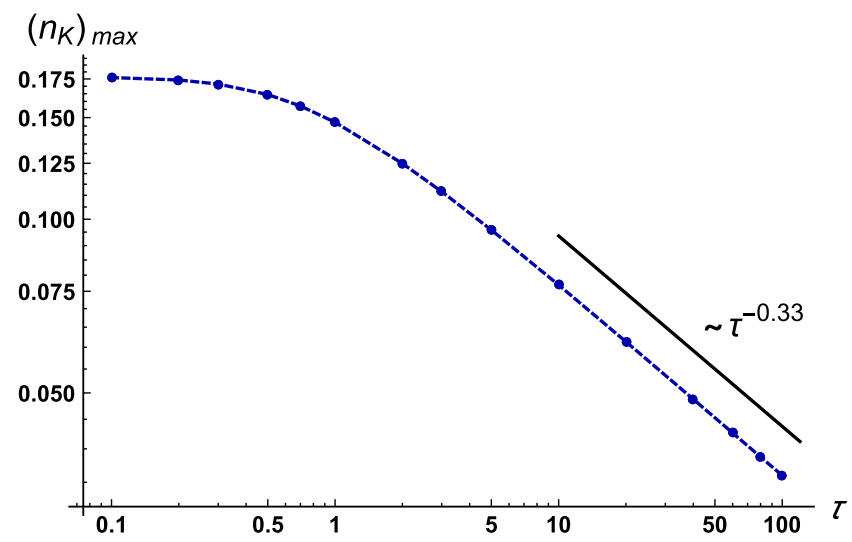

FIG. 4. Log-Log plot of the maximum average kink density $\left(n_{K}\right)_{\max }$ versus $\tau$. For larger values of $\tau$, the power law manifested is $\sim \tau^{-0.33}$.

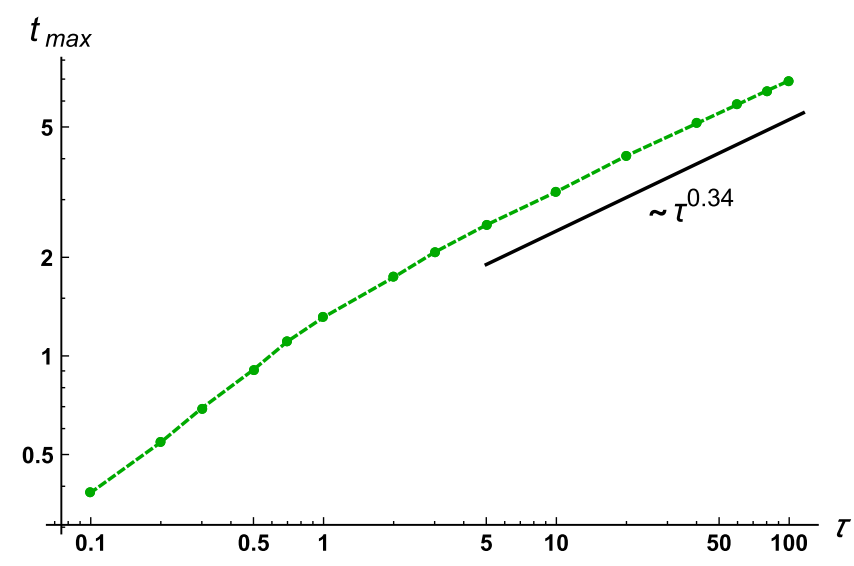

FIG. 5. Log-Log plot of the time at which maximum average kink density $\left(n_{K}\right)_{\max }$ occurs versus $\tau$. For larger values of $\tau$, the power law exhibited is $\sim \tau^{0.34}$. faster the phase transition (smaller $\tau$ ), the more kinks are produced and the quicker they start annihilating.

\section{CONCLUSIONS}

Our key result is Eq. (33). It tells us how the quantum vacuum breaks up into classical solitons after a quench. Further, it shows that the result at late times is universal and does not depend on the quench timescale, even though there is some dependence closer to the time of the phase transition. In the Kibble-Zurek proposal for kinks produced during a thermal phase transition, the kink density immediately after the phase transition depends on the quench timescale and is proportional to $\tau^{-1 / 4}$ in certain systems [24]. This is to be contrasted with the $\tau^{-1 / 3}$ falloff in Fig. 4.

We have also cross-checked our results by numerically estimating $n_{K}$ as the inverse of the correlation length that is extracted from the covariance matrix $K$, as well as analytically by studying the limiting case of a sudden phase transition with $\tau=0$. We plan to present these crosschecks in a separate publication [25]. Similar analyses for a sudden $(\tau=0)$ thermal quench have been done in $[26,27]$ by different techniques; a $t^{-1 / 2}$ scaling of defect density was also observed, but the dependence on quench timescale was not studied.

In our approach we have taken vanishing interaction strength $\lambda=0$, and it is of interest to ask how the results might change if $\lambda$ is different from zero. As we have noted in the Introduction, our result for the number density of kinks can be thought of as the $\mathcal{O}\left(\lambda^{0}\right)$ term in a perturbation expansion in $\lambda$. It should be possible to compute higherorder corrections in $\lambda$ using perturbation theory. In particular, there will be $\lambda$-dependent corrections to our wave functional in (13). However the corrections to the number density of kinks also depend on the parameter $\tau$ that determines how quickly the potential for the field $\phi$ changes. If $\tau$ is very small, the wave functional can be computed in the "sudden approximation" [25], which will be valid if the timescale for changes in the potential is much shorter than the timescale set by the interaction term.

Our analysis also suggests that the interaction between kinks is not important for sudden phase transitions. As can be seen in Fig. 4, the maximum kink number density for $\tau=0$ is $\left(n_{K}\right)_{\max } \approx 0.175$, and the average kink separation $n_{K}^{-1}$ is greater than at least $\sim 6$ times the width of the kink. Since the attractive kink-antikink force decreases exponentially with distance in $1+1$ dimensions, the effect of interkink forces can be consistently disregarded.

The analysis we have done in this paper can be generalized to higher dimensions to discuss global vortex formation in two spatial dimensions and global monopole formation in three spatial dimensions, since the models for these can be truncated to free fields in time-dependent backgrounds [25]. The introduction of gauge fields, however, will lead to new interactions that will be more difficult to analyze. 
Finally, it is worth mentioning that there is a deeper foundational question in this problem that we have studied. Our initial state is a translationally invariant quantum vacuum, while the final state involves classical kinks with definite positions and velocities. The translational symmetry is preserved when averages are taken over an ensemble of kink realizations, but each realization of the kinks breaks translational symmetry. As in Schrödinger's cat, the classical kinks materialize and break translational symmetry only when there is a detector that detects them.

\section{ACKNOWLEDGMENTS}

We thank Dan Boyanovsky for comments. M. M. is supported by the National Science Foundation Grants No. PHY-1613708 and No. PHY-2012195. T. V. is supported by the U.S. Department of Energy, Office of High Energy Physics, under Award No. DE-SC0019470 at Arizona State University. G. Z. is supported by Moogsoft and the Foundational Questions Institute (FQXi).

\section{APPENDIX A: SOME PROPERTIES OF CIRCULANT MATRICES}

A generic $N \times N$ matrix $A=\left(a_{i j}\right)$ is circulant when its entries $a_{i j}$ only depend on $j-i(\bmod N)$. In other words, $a_{i j} \equiv a_{j-i(\bmod N)}$. It is useful to introduce the permutation matrix

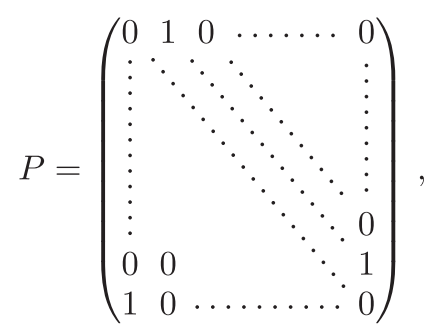

verifying $P^{-1}=P^{T}=P^{N-1}$. The matrix $A$ is seen to be a degree $N-1$ polynomial in $P$,

$$
A=a_{0} I+a_{1} P+a_{2} P^{2}+\cdots+a_{N-1} P^{N-1} .
$$

This immediately implies that sums, products, transposes, and Hermitian conjugates of circulant matrices are also circulant (and that circulant matrices commute with each other). An additional consequence is that bilinears involving circulant matrices are unchanged under circular permutations of vector elements. Indeed, given a positive integer $n \leq N-1$ and two vectors $X$ and $Y$, we have

$$
\left(P^{n} X\right)^{T} A\left(P^{n} Y\right)=X^{T} P^{-n} A P^{n} Y=X^{T} A Y .
$$

Moreover the circulant matrix $A$ can be diagonalized via the unitary discrete Fourier transform matrix $F=\left(f_{j k}\right)$, where $f_{j k} \equiv \frac{1}{\sqrt{N}} e^{-i 2 \pi j k / N}$,

$$
A=F^{-1} D F=F^{\dagger} D F .
$$

This shows that if a circulant matrix is invertible, then its inverse is also circulant. All these properties are often used in the main text and, in particular, when using the fact that $\left\langle\operatorname{sgn}\left(\hat{\phi}_{i} \hat{\phi}_{i+1}\right)\right\rangle=\left\langle\operatorname{sgn}\left(\hat{\phi}_{1} \hat{\phi}_{2}\right)\right\rangle$ in the lead up to Eq. (16).

\section{APPENDIX B: TWO-DIMENSIONAL GAUSSIAN INTEGRAL OVER THE FIRST QUADRANT}

The derivation of the two-dimensional Gaussian integral over the first quadrant $x, y \geq 0$ follows from the change of variables $x=s y$,

$$
\begin{aligned}
\int_{0}^{\infty} & d x \int_{0}^{\infty} d y e^{-\frac{1}{2}\left(a x^{2}+2 b x y+c y^{2}\right)} \\
= & \int_{0}^{\infty} d s \int_{0}^{\infty} d y y e^{-\frac{y^{2}}{2}\left(a s^{2}+2 b s+c\right)} \\
& =\int_{0}^{\infty} \frac{d s}{a s^{2}+2 b s+c} \\
& =\frac{1}{\sqrt{a c-b^{2}}}\left[\frac{\pi}{2}-\tan ^{-1}\left(\frac{b}{\sqrt{a c-b^{2}}}\right)\right] .
\end{aligned}
$$

Here we have assumed that $a, b$, and $c$ are real numbers such that $a>0$ and $a c-b^{2}>0$. This ensures that all expressions in the above equations are well posed. This identity is used in the main text in the lead up to Eq. (21).

\section{APPENDIX C: SOME PROPERTIES OF BLOCK MATRICES}

Consider a square matrix $M$ partitioned into four blocks of arbitrary size as follows:

$$
M=\left(\begin{array}{ll}
A & B \\
C & D
\end{array}\right) .
$$

Performing the analog of a LDU decomposition on this block matrix, we can write

$$
\begin{aligned}
M & =\left(\begin{array}{ll}
A & B \\
C & D
\end{array}\right) \\
& =\left(\begin{array}{cc}
I & B D^{-1} \\
0 & I
\end{array}\right)\left(\begin{array}{cc}
A-B D^{-1} C & 0 \\
0 & D
\end{array}\right)\left(\begin{array}{cc}
I & 0 \\
D^{-1} C & I
\end{array}\right) .
\end{aligned}
$$

Here we have assumed that $A$ and $D$ are square invertible matrices. Note that the identity matrices can have different sizes. This expression immediately implies that

$$
\operatorname{det} M=\operatorname{det}\left(A-B D^{-1} C\right) \operatorname{det} D .
$$

The matrix $A-B D^{-1} C$ is called the Schur complement of $D$. This formula is used in the main text in the lead up to Eq. (23). Assuming invertibility of the Schur complement, we can also write 


$$
\begin{aligned}
M^{-1} & =\left(\begin{array}{cc}
I & 0 \\
-D^{-1} C & I
\end{array}\right)\left(\begin{array}{rr}
\left(A-B D^{-1} C\right)^{-1} & 0 \\
0 & D^{-1}
\end{array}\right)\left(\begin{array}{cc}
I & -B D^{-1} \\
0 & I
\end{array}\right) \\
& =\left(\begin{array}{cc}
\left(A-B D^{-1} C\right)^{-1} & -\left(A-B D^{-1} C\right)^{-1} B D^{-1} \\
-D^{-1} C\left(A-B D^{-1} C\right)^{-1} & D^{-1}+D^{-1} C\left(A-B D^{-1} C\right)^{-1} B D^{-1}
\end{array}\right),
\end{aligned}
$$

which allows one to directly read off the inverse of the Schur complement of $D$ on the block decomposition of $M^{-1}$. This identity is used in the main text in the lead up to Eq. (25).

[1] T. Kibble, Topology of cosmic domains and strings, J. Phys. A 9, 1387 (1976).

[2] T. Kibble, Some implications of a cosmological phase transition, Phys. Rep. 67, 183 (1980).

[3] W. Zurek, Cosmological experiments in superfluid helium? Nature (London) 317, 505 (1985).

[4] W. Zurek, Cosmic strings in laboratory superfluids and the topological remnants of other phase transitions, Acta Phys. Pol. B 24, 1301 (1993).

[5] W. Zurek, Cosmological experiments in condensed matter systems, Phys. Rep. 276, 177 (1996).

[6] G. Karra and R. Rivers, Initial vortex densities after a quench, Phys. Lett. B 414, 28 (1997).

[7] T. Vachaspati, Kinks and Domain Walls: An Introduction to Classical and Quantum Solitons (Cambridge University Press, Cambridge, England, 2010).

[8] T. Kibble, Phase-transition dynamics in the lab and the universe, Phys. Today 60, no. 9, 47 (2007).

[9] I. Chuang, R. Durrer, N. Turok, and B. Yurke, Cosmology in the laboratory: Defect dynamics in liquid crystals, Science 251, 1336 (1991).

[10] M. J. Bowick, L. Chandar, E. A. Schiff, and A. M. Srivastava, The cosmological Kibble mechanism in the laboratory: String formation in liquid crystals, Science 263, 943 (1994).

[11] P. C. Hendry, N. S. Lawson, R. A. M. Lee, P. V. E. McClintock, and C. D. H. Williams, Generation of defects in superfluid the as an analogue of the formation of cosmic strings, Nature (London) 368, 315 (1994).

[12] M. E. Dodd, P. C. Hendry, N. S. Lawson, P. V. E. McClintock, and C.D.H. Williams, Nonappearance of Vortices in Fast Mechanical Expansions of Liquid ${ }^{4} \mathrm{He}$ through the Lambda Transition, Phys. Rev. Lett. 81, 3703 (1998).

[13] V. M. H. Ruutu, V. B. Eltsov, A. J. Gill, T. W. B. Kibble, M. Krusius, Y. G. Makhlin, B. Plaais, G. E. Volovik, and $\mathrm{W}$. $\mathrm{Xu}$, Vortex formation in neutron-irradiated superfluid 3he as an analogue of cosmological defect formation, Nature (London) 382, 334 (1996).

[14] C. Buerle, Y. M. Bunkov, S. N. Fisher, H. Godfrin, and G. R. Pickett, Laboratory simulation of cosmic string formation in the early universe using superfluid 3he, Nature (London) 382, 332 (1996).

[15] V. Eltsov, M. Krusius, and G. Volovik, Vortex Formation and Dynamics in Superfluid 3he and Analogies in Quantum Field Theory (Elsevier, New York, 2005), pp. 1-137.

[16] R. Monaco, J. Mygind, and R. J. Rivers, Zurek-Kibble Domain Structures: The Dynamics of Spontaneous Vortex Formation in Annular Josephson Tunnel Junctions, Phys. Rev. Lett. 89, 080603 (2002).

[17] R. Carmi, E. Polturak, and G. Koren, Observation of Spontaneous Flux Generation in a Multi-JosephsonJunction Loop, Phys. Rev. Lett. 84, 4966 (2000).

[18] A. Maniv, E. Polturak, and G. Koren, Observation of Magnetic Flux Generated Spontaneously during a Rapid Quench of Superconducting Films, Phys. Rev. Lett. 91, 197001 (2003).

[19] S. Ducci, P. L. Ramazza, W. González-Viñas, and F. T. Arecchi, Order Parameter Fragmentation after a SymmetryBreaking Transition, Phys. Rev. Lett. 83, 5210 (1999).

[20] J. Beugnon and N. Navon, Exploring the Kibble-Zurek mechanism with homogeneous Bose gases, J. Phys. B 50, 022002 (2017).

[21] T. Vachaspati and G. Zahariade, Classical-quantum correspondence for fields, J. Cosmol. Astropart. Phys. 09 (2019) 015.

[22] J. Olle, O. Pujolas, T. Vachaspati, and G. Zahariade, Quantum evaporation of classical breathers, Phys. Rev. D 100, 045011 (2019).

[23] M. Mukhopadhyay and T. Vachaspati, Rolling classical scalar field in a linear potential coupled to a quantum field, Phys. Rev. D 100, 096018 (2019).

[24] P. Laguna and W. H. Zurek, Density of Kinks after a Quench: When Symmetry Breaks, How Big Are the Pieces? Phys. Rev. Lett. 78, 2519 (1997).

[25] M. Mukhopadhyay, T. Vachaspati, and G. Zahariade (to be published).

[26] D. Boyanovsky, Quantum spinodal decomposition, Phys. Rev. E 48, 767 (1993).

[27] D. Boyanovsky, H. de Vega, and R. Holman, Nonequilibrium phase transitions in condensed matter and cosmology: Spinodal decomposition, condensates and defects, NATO Sci. Ser. C 549, 139 (2000). 